\title{
Milnor fibers and Links of Local Complete Intersections
}

\author{
David B. Massey
}

\begin{abstract}
We discuss and prove a number of cohomological results for Milnor fibers, real links, and complex links of local complete intersections with singularities of arbitrary dimension.
\end{abstract}

\section{Introduction}

Suppose that $X$ is a purely $(n+1)$-dimensional local complete intersection. For convenience, we will assume that $X$ is locally embedded in an open subset $\mathcal{U}$ of $\mathbb{C}^{N}$, and that $\mathbf{0} \in X$. We will assume that $\mathbf{0}$ is a singular point of $X$. Suppose that we have a complex analytic function $\hat{f}:(\mathcal{U}, \mathbf{0}) \rightarrow(\mathbb{C}, 0)$, and let $f$ denote the restriction of $\hat{f}$ to $X$.

We are interested in what one can say about standard topological objects - like real links, complex links, and Milnor fibers (see Section 2 for definitions) - in the case where the dimension of the singular set of $X$, $\Sigma X$, and the dimension of the critical locus of $f, \Sigma f$, are arbitrary. Of course, part of the issue is that we must define what notion of "critical locus" we are using for a function on a space with possibly non-isolated singularities.

We let $d:=\operatorname{dim}_{0} \Sigma X$. If $d=0$, we define $d_{f}:=0$; otherwise, we define $d_{f}:=\operatorname{dim}_{0} \overline{\Sigma X \backslash V(f)}$. We are interested in points which are "cohomological critical points of $f$ ", that is, points where the reduced cohomology of the Milnor fiber is not zero. Therefore, we let $F_{f-f(\mathbf{x}), \mathbf{x}}$ denote the compact Milnor fiber of $f-f(\mathbf{x})$ at $\mathbf{x}$, and define:

Definition 1.1. The $\mathbb{Z}$-critical locus of $f, \Sigma_{\mathbb{Z}} f$, is the set

$$
\left\{\mathbf{x} \in X \mid \widetilde{H}^{*}\left(F_{f-f(\mathbf{x}), \mathbf{x}} ; \mathbb{Z}\right) \neq 0\right\} .
$$

It is well-known that, given any Whitney stratification of $X$, the $\mathbb{Z}$-critical locus is contained in the stratified critical locus of $f$. In addition, stratified critical values are local isolated. Consequently, by taking a smaller neighborhood of the origin, if necessary, we may assume that $\Sigma_{\mathbb{Z}} f \subseteq V(f)$. We let $s_{f}:=\operatorname{dim}_{0} \Sigma_{\mathbb{Z}} f$.

Milnor fibers and the real link of a hypersurface singularity have been fundamental objects of study since Milnor's book [14] on the subject appeared in 1968. For complete intersections with isolated singular points, the Milnor fiber and the real link were investigated by Looijenga in his book [10] from 1984. The complex link was studied in depth by Lê and Kato in 1975 in [9], and for complete intersections by Lê in 1979 in [8]. The complex link, and its relationship to the real link, was discussed at length in Goresky and MacPherson's 1988 book "Stratified Morse Theory" [3].

There are several known results in the general situation that we are in. Note that we always use $\mathbb{Z}$ coefficients in this paper when dealing with ordinary cohomology.

AMS subject classifications: 32B15, 32C35, 32C18, 32B10.

Keywords: Milnor fiber, real link, complex link, local complete intersection 
- In [4], Hamm generalizes a result of Milnor for hypersurfaces and proves that the real link of $X$ at $\mathbf{0}$, $K_{X, \mathbf{0}}$, is $(n-1)$-connected, and so, in particular, that $\widetilde{H}^{k}\left(K_{X, \mathbf{0}}\right)=0$ for $k \leq n-1$.

- In [8], Lê proves that the complex link of $X$ at $\mathbf{0}, \mathbb{L}_{X, \mathbf{0}}$, has the homotopy-type of a finite bouquet of $n$-spheres and so, in particular, that $\widetilde{H}^{k}\left(\mathbb{L}_{X, \mathbf{0}}\right)=0$ for $k \neq n$.

- Lê's result on the complex link easily extends to the links of all strata (see Section 2) of any Whitney stratification of $X$. This implies that the shifted constant sheaf $\mathbb{Z}_{X}^{\bullet}[n+1]$ is a perverse sheaf. Then, the general results that the shifted nearby and vanishing cycles of a perverse sheaf are perverse imply that $\widetilde{H}^{k}\left(F_{f, \mathbf{0}}\right)=0$ unless $n-s_{f} \leq k \leq n$, and $H^{k}\left(F_{f, \mathbf{0}}, \partial F_{f, \mathbf{0}}\right)=0$ unless $n \leq k \leq 2 n$.

- In Chapter 6 of [15], Schürmann investigates similar questions by similar techniques, but does not obtain most of our general results.

Notice how none of the results above detect the singular set of $X$ itself; they are independent of the values of $d$ and $d_{f}$. We want to look at cohomological results that actually notice that $X$ may be very singular.

In this paper, we prove:

1. $H^{k}\left(F_{f, \mathbf{0}}, \partial F_{f, \mathbf{0}}\right)=0$ unless $k=2 n$ or $n \leq k \leq n+\max \left\{s_{f}, d_{f}\right\}$.

2. $H^{k}\left(\mathbb{L}_{X, \mathbf{0}}, \partial \mathbb{L}_{X, \mathbf{0}}\right)=0$ unless $k=2 n$ or $n \leq k \leq n+d$.

3. There is a (dual) Wang exact sequence

$$
\begin{aligned}
\cdots \rightarrow H^{k-1}\left(F_{f, \mathbf{0}}, \partial F_{f, \mathbf{0}}\right) \rightarrow H^{k}\left(K_{X, \mathbf{0}}, K_{V(f), \mathbf{0}}\right) \rightarrow H^{k}\left(F_{f, \mathbf{0}}, \partial F_{f, \mathbf{0}}\right) \stackrel{\text { id }-T_{f, \mathbf{0}}}{\longrightarrow} \\
\quad H^{k}\left(F_{f, \mathbf{0}}, \partial F_{f, \mathbf{0}}\right) \rightarrow H^{k+1}\left(K_{X, \mathbf{0}}, K_{V(f), \mathbf{0}}\right) \rightarrow \cdots,
\end{aligned}
$$

where $T_{f, 0}$ is the monodromy automorphism on the Milnor fiber with boundary. In particular, $H^{k}\left(K_{X, \mathbf{0}}, K_{V(f), \mathbf{0}}\right)=0$ unless $k=2 n, 2 n+1$ or $n \leq k \leq n+\max \left\{s_{f}, d_{f}\right\}+1$.

4. If $H$ is a generic hyperplane in the ambient space $\mathcal{U}$, then $H^{k}\left(K_{X, \mathbf{0}}, K_{X \cap H, \mathbf{0}}\right)=0$ unless $k=2 n, 2 n+1$ or $n \leq k \leq n+d+1$.

5. Suppose that $n \geq 1$ and $s_{f}=0$. Then, there is well-defined variation map

$$
\operatorname{var}: H^{n}\left(F_{f, \mathbf{0}}\right) \rightarrow H^{n}\left(F_{f, \mathbf{0}}, \partial F_{f, \mathbf{0}}\right)
$$

and an exact sequence

$$
0 \rightarrow H^{n}\left(K_{X, \mathbf{0}}\right) \rightarrow H^{n}\left(F_{f, \mathbf{0}}\right) \stackrel{\mathrm{var}}{\longrightarrow} H^{n}\left(F_{f, \mathbf{0}}, \partial F_{f, \mathbf{0}}\right) \rightarrow H^{n+1}\left(K_{X, \mathbf{0}}\right) \rightarrow 0 .
$$

In particular, $H^{n}\left(K_{X, \mathbf{0}}\right)$ injects into $H^{n}\left(F_{f, \mathbf{0}}\right)$, and var is an isomorphism if and only if $H^{n}\left(K_{X, \mathbf{0}}\right)=$ $H^{n+1}\left(K_{X, \mathbf{0}}\right)=0$. Furthermore, for all $k \geq n+1, H^{k}\left(F_{f, \mathbf{0}}, \partial F_{f, \mathbf{0}}\right) \cong H^{k+1}\left(K_{X, \mathbf{0}}\right)$.

6. Suppose that $n \geq 1$ and $s_{f}=0$. Then, for all $k \geq n+1, H^{k}\left(F_{f, \mathbf{0}}, \partial F_{f, \mathbf{0}}\right) \cong H^{k+1}\left(K_{X, \mathbf{0}}\right)$, and so is independent of $f$. In particular, for all $k \geq n+1$,

$$
H^{k}\left(F_{f, \mathbf{0}}, \partial F_{f, \mathbf{0}}\right) \cong H^{k+1}\left(K_{X, \mathbf{0}}\right) \cong H^{k}\left(\mathbb{L}_{X, \mathbf{0}}, \partial \mathbb{L}_{X, \mathbf{0}}\right) .
$$

7. $H^{n}\left(K_{X, \mathbf{0}}\right)$ injects into $H^{n}\left(\mathbb{L}_{X, \mathbf{0}}\right)$. 
8. $H^{k}\left(K_{X, \mathbf{0}}\right)=0$, unless $k=0, k=2 n+1$, or $n \leq k \leq n+d+1$.

Most of the results above are known in the case where $d=0$, i.e., where $X$ is an isolated complete intersection singularity; in that case, the Milnor fibers, real links, and complex links are all smooth and one can use Poincaré-Lefschetz duality. However, when $d \geq 1$, the Milnor fiber, real link and complex link are all singular, and one cannot use Poincaré-Lefschetz duality. However, Verdier duality is still at our disposal, and leads to all of these new general results.

\section{Standard definitions and a classical result}

We must discuss some basic background on Whitney stratifications, Milnor fibrations, real and complex links, the derived category of complexes of sheaves of $\mathbb{Z}$-modules, and perverse sheaves. General references on stratifications, links, and Milnor fibrations are [3], [14], [1], and [7].

Suppose that $\mathcal{U}$ is an open subset of $\mathbb{C}^{N}$, that $X$ is a complex analytic subset of $\mathcal{U}$, and that $\mathbf{p} \in X$. Suppose that $f:(X, \mathbf{p}) \rightarrow(\mathbb{C}, 0)$ is a complex analytic function.

Then, the following definitions are standard:

\section{Definition 2.1.}

Let $B_{\epsilon}(\mathbf{p})$ be a sufficiently small closed ball of radius $\epsilon>0$, with boundary $S_{\epsilon}(\mathbf{p})$. Let $L: \mathbb{C}^{N} \rightarrow \mathbb{C}^{\text {be a }}$ generic affine linear form with $L(\mathbf{p})=0$. Finally, let $\eta \in \mathbb{C}$ be such that $0<|\eta| \ll \epsilon$.

Then, the homeomorphism-types of the following spaces are independent of the choices of $\epsilon$, $\eta$, and $L$ :

1. the real link of $X$ at $\mathbf{p}$ is $K_{X, \mathbf{p}}:=S_{\epsilon}(\mathbf{p}) \cap X$;

2. the (compact) Milnor fiber of $f$ at $\mathbf{p}$ is $F_{f, \mathbf{p}}:=B_{\epsilon}(\mathbf{p}) \cap f^{-1}(\eta)$;

3. the boundary of the the Milnor fiber of $f$ at $\mathbf{p}$ is $\partial F_{f, \mathbf{p}}:=S_{\epsilon}(\mathbf{p}) \cap f^{-1}(\eta)$;

4. the (compact) complex link of $X$ at $\mathbf{p}$ is $\mathbb{L}_{X, \mathbf{p}}:=B_{\epsilon}(\mathbf{p}) \cap X \cap L^{-1}(\eta)=F_{L_{\mid X}}, \mathbf{p}$; and

5. the boundary of the complex link of $X$ at $\mathbf{p}$ is $\partial \mathbb{L}_{X, \mathbf{p}}:=S_{\epsilon}(\mathbf{p}) \cap X \cap L^{-1}(\eta)=\partial F_{L_{\left.\right|_{X}}, \mathbf{p}}$.

If we replace the closed ball with the open ball, the homotopy-types of the resulting non-compact Milnor fiber and complex link are the same as those of the compact ones.

Furthermore, if we let $H:=V(L)$, then $\partial \mathbb{L}_{X, \mathbf{p}}$ is homeomorphic to $S_{\epsilon}(\mathbf{p}) \cap X \cap H=K_{X \cap H, \mathbf{p}}$.

We also need to define complex links of Whitney strata. Throughout this paper, our Whitney strata are always assumed to be connected.

Definition 2.2. Suppose that $\mathfrak{S}$ is a complex analytic Whitney stratification of $X$. Let $S \in \mathfrak{S}$, let $\mathbf{p} \in S$, and let $M$ be a complex submanifold of $\mathcal{U}$ of complementary dimension (i.e., of dimension $N-\operatorname{dim} S$ ), which transversely intersects $S$ at $\mathbf{p}$.

Then, $M$ is a normal slice to $S$ at $\mathbf{p}$ and, for sufficiently small $\epsilon>0$, the homeomorphism-type of the pair

$$
\left(B_{\epsilon}(\mathbf{p}) \cap X \cap M, \mathbb{L}_{X \cap M, \mathbf{p}}\right)
$$


is independent of the choice of $M$ and $\mathbf{p}$.

This homeomorphism-type is the normal Morse data of $S$ in $X$ and the homeomorphism-type of $\mathbb{L}_{X \cap M, \mathbf{p}}$ is the complex link of $S$ in $X$. We denote this pair by $\left(\mathbb{N}_{X, S}, \mathbb{L}_{X, S}\right)$.

We need the fundamental result of Lê from [8] on local complete intersections.

Theorem 2.3. (Lê, 1979) Suppose that $X$ is a local complete intersection of pure dimension $(n+1)$ and that $\mathfrak{S}$ is a Whitney stratification of $X$. Let $S \in \mathfrak{S}$.

Then, $\mathbb{L}_{X, S}$ has the homotopy-type of a finite bouquet of spheres of dimension $n-\operatorname{dim} S$. In particular, $\widetilde{H}^{k}\left(\mathbb{L}_{X, S} ; \mathbb{Z}\right)$ is zero if $k \neq n-\operatorname{dim} S$ and

$$
\widetilde{H}^{n-\operatorname{dim} S}\left(\mathbb{L}_{X, S} ; \mathbb{Z}\right) \cong \mathbb{Z}^{m}
$$

for some finite value of $m$ (possibly 0$)$.

\section{Background on the derived category}

We continue as before with $\mathcal{U}$ being an open subset of $\mathbb{C}^{N}$, and $X$ being a complex analytic subset of $\mathcal{U}$. We now want to see what we get by using the machinery of the derived category, perverse sheaves, nearby cycles, and vanishing cycles. As general references for the derived category and perverse sheaves, we recommend [5] and [2].

As we are interested in results on integral cohomology, throughout this paper, our complexes of sheaves $\mathbf{A}^{\bullet}$ will be bounded, constructible sheaves of $\mathbb{Z}$-modules on $X$; we write $\mathbf{A}^{\bullet} \in D_{c}^{b}(X)$.

\section{Morse modules and perverse sheaves}

We first need a cohomological version of a normal Morse data to strata with coefficients in a complex of sheaves $\mathbf{A}^{\bullet}$.

Definition 3.1. Suppose that $\mathbf{A}^{\bullet}$ is a bounded complex of sheaves of $\mathbb{Z}$-modules, which is constructible with respect to a Whitney stratification $\mathfrak{S}$. Let $S \in \mathfrak{S}$.

Then, using our notation from the previous section, the isomorphism-type of the hypercohomology

$$
m_{S}^{k}\left(\mathbf{A}^{\bullet}\right):=\mathbb{H}^{k-\operatorname{dim} S}\left(\mathbb{N}_{X, S}, \mathbb{L}_{X, S} ; \mathbf{A}^{\bullet}\right)
$$

is well-defined (i.e., is independent of the choices made in defining the normal Morse data). We call this the degree $k$ Morse module of $S$ with respect to $\mathbf{A}^{\bullet}$. The Morse modules are necessarily finitely-generated $\mathbb{Z}$-modules.

In particular,

$$
\begin{aligned}
& m_{S}^{k}\left(\mathbb{Z}_{X}^{\bullet}[n+1]\right)=\mathbb{H}^{k-\operatorname{dim} S}\left(\mathbb{N}_{X, S}, \mathbb{L}_{X, S} ; \mathbb{Z}_{X}^{\bullet}[n+1]\right) \cong \\
& H^{n+1+k-\operatorname{dim} S}\left(\mathbb{N}_{X, S}, \mathbb{L}_{X, S} ; \mathbb{Z}\right) \cong \widetilde{H}^{n+k-\operatorname{dim} S}\left(\mathbb{L}_{X, S} ; \mathbb{Z}\right)
\end{aligned}
$$


We do not wish to define perverse sheaves here, and we are not going to list all of the well-known results on perverse sheaves that we use in this section. However, we do want to state a fundamental result gives a characterization of perverse sheaves, a result which may not be so well-known.

Theorem 3.2. ([5], Theorem 10.3.2) A bounded constructible complex of sheaves $\mathbf{P} \bullet$ is perverse if and only if all of the Morse modules of $\mathbf{P}^{\bullet}$ are concentrated in degree zero, i.e., are zero in all non-zero degrees.

This is independent of the Whitney stratification, with respect to which $\mathbf{P}^{\bullet}$ is constructible, that is used in defining the Morse modules.

In light of Theorem 3.2, the result of Lê in Theorem 2.3 tells us:

Theorem 3.3. Suppose that $X$ is a local complete intersection of pure dimension $(n+1)$. Then, $\mathbb{Z}_{X}^{\bullet}[n+1]$ is a perverse sheaf.

\section{Stalk and costalk cohomology}

We are interested in critical points of complex analytic functions $f: X \rightarrow \mathbb{C}$. Let $j_{f}$ denote the inclusion of $V(f)$ into $X$. For all $\mathbf{x} \in X$, let $m_{\mathbf{x}}$ denote the inclusion of $\{\mathbf{x}\}$ into $X$ and, for all $\mathbf{x} \in V(f)$, let $\hat{m}_{\mathbf{x}}$ denote the inclusion of $\{\mathbf{x}\}$ into $V(f)$, so that $m_{\mathbf{x}}=j_{f} \circ \hat{m}_{\mathbf{x}}$. For $\mathbf{A}^{\bullet} \in \mathbb{D}_{c}^{b}(X)$, the stalk cohomology of $\mathbf{A}^{\bullet}$ at $x$ is

$$
H^{k}\left(\mathbf{A}^{\bullet}\right)_{\mathbf{x}}=H^{k}\left(m_{\mathbf{x}}^{*} \mathbf{A}^{\bullet}\right) \cong \mathbb{H}^{k}\left(B_{\epsilon}(\mathbf{x}) ; \mathbf{A}^{\bullet}\right),
$$

for sufficiently small $\epsilon>0$; the costalk cohomology of $\mathbf{A}^{\bullet}$ at $x$ is

$$
H^{k}\left(m_{\mathbf{x}} \mathbf{A}^{\bullet}\right) \cong \mathbb{H}^{k}\left(B_{\epsilon}(\mathbf{x}), B_{\epsilon}(\mathbf{x})-\{\mathbf{x}\} ; \mathbf{A}^{\bullet}\right) \cong \mathbb{H}^{k}\left(B_{\epsilon}(\mathbf{x}), S_{\epsilon}(\mathbf{x}) ; \mathbf{A}^{\bullet}\right),
$$

for sufficiently small $\epsilon>0$.

If $\mathbf{P}^{\bullet}$ is a perverse sheaf on a space $X$, which is supported on an $r$-dimensional subset of $X$, and $\mathbf{x} \in X$, then:

1. $H^{k}\left(m_{\mathbf{x}}^{*} \mathbf{A}^{\bullet}\right)=0$, unless $-r \leq k \leq 0$, and

2. $H^{k}\left(m_{\mathbf{x}}^{!} \mathbf{A}^{\bullet}\right)=0$, unless $0 \leq k \leq r$.

\section{Nearby and vanishing cycles, the Milnor fiber, and the monodromy}

We remind the reader that that the shifted nearby and vanishing cycle functors, $\psi_{f}[-1]$ and $\phi_{f}[-1]$, take perverse sheaves on $X$ to perverse sheaves on $V(f)$, and their stalk cohomology gives the cohomology and "reduced" cohomology of the Milnor of $f$. To be precise,

$$
H^{k}\left(\psi_{f}[-1] \mathbf{A}^{\bullet}\right)_{\mathbf{x}} \cong H^{k}\left(\hat{m}_{\mathbf{x}} \psi_{f}[-1] \mathbf{A}^{\bullet}\right) \cong \mathbb{H}^{k-1}\left(F_{f, \mathbf{x}} ; \mathbf{A}^{\bullet}\right)
$$

and

$$
H^{k}\left(\phi_{f}[-1] \mathbf{A}^{\bullet}\right)_{\mathbf{x}} \cong H^{k}\left(\hat{m}_{\mathbf{x}} \phi_{f}[-1] \mathbf{A}^{\bullet}\right) \cong \mathbb{H}^{k}\left(B_{\epsilon}(\mathbf{x}) \cap X, F_{f, \mathbf{x}} ; \mathbf{A}^{\bullet}\right)
$$


Note that, if $\mathbf{A}^{\bullet}$ is not the constant sheaf (or some shift of it), one must take care in thinking of the vanishing cycle cohomology as the "reduced" cohomology; it will not be as trivial as removing a copy of $\mathbb{Z}$ in one degree. Note, also, that we use the standard definition of the vanishing cycles; the one used in [5] is shifted by one from this.

The costalk cohomology of the nearby cycles gives the cohomology of the Milnor fiber modulo its boundary; that is, for all $\mathbf{x} \in V(f)$,

$$
H^{k}\left(\hat{m}_{\mathbf{x}}^{!} \psi_{f}[-1] \mathbf{A}^{\bullet}\right)_{\mathbf{x}} \cong \mathbb{H}^{k-1}\left(F_{f, \mathbf{x}}, \partial F_{f, \mathbf{x}} ; \mathbf{A}^{\bullet}\right) .
$$

There is a natural map between functors $\hat{m}_{\mathbf{x}}^{!} \stackrel{\tau_{\mathbf{x}}}{\longrightarrow} \hat{m}_{\mathbf{x}}^{*}$. On cohomology, this yields the map induced by the inclusion of pairs $\left(F_{f, \mathbf{x}}, \emptyset\right) \hookrightarrow\left(F_{f, \mathbf{x}}, \partial F_{f, \mathbf{x}}\right)$ :

$$
\mathbb{H}^{k-1}\left(F_{f, \mathbf{x}}, \partial F_{f, \mathbf{x}} ; \mathbf{A}^{\bullet}\right) \rightarrow \mathbb{H}^{k-1}\left(F_{f, \mathbf{x}} ; \mathbf{A}^{\bullet}\right) .
$$

There are monodromy automorphisms in the derived category, induced by letting the value of $f$ travel once, counterclockwise, around a small circle:

$$
T_{f}: \psi_{f}[-1] \mathbf{A}^{\bullet} \rightarrow \psi_{f}[-1] \mathbf{A}^{\bullet} \quad \text { and } \quad \widetilde{T}_{f}: \phi_{f}[-1] \mathbf{A}^{\bullet} \rightarrow \phi_{f}[-1] \mathbf{A}^{\bullet} .
$$

On the stalk cohomology of the nearby cycles, $\hat{m}_{\mathbf{x}}^{*} T_{f}$ induces the usual monodromy automorphism on $\mathbb{H}^{*}\left(F_{f, \mathbf{x}} ; \mathbf{A}^{\bullet}\right)$. On the costalk cohomology of the nearby cycles, $\hat{m}_{\mathbf{x}}^{!} T_{f}$ induces the usual monodromy automorphism on $\mathbb{H}^{*}\left(F_{f, \mathbf{x}}, \partial F_{f, \mathbf{x}} ; \mathbf{A}^{\bullet}\right)$.

The following theorem is immediate from [12], Theorems 3.3 and 3.5.

Theorem 3.4. If the Morse modules of $\mathbf{A}^{\bullet}$ are all free Abelian, then so are all of the Morse modules of $\psi_{f}[-1] \mathbf{A}^{\bullet}$ and $\phi_{f}[-1] \mathbf{A}^{\bullet}$.

There are two standard natural distinguished triangles, and their duals, related to the Milnor fibration. We write these triangles in their in-line forms. We remind the reader that $j_{f}$ denotes the closed inclusion of $V(f)$ into $X$, and we let $i_{f}$ denote the open inclusion of $X-V(f)$ into $X$.

The vanishing triangle:

$$
\rightarrow j_{f}^{*}[-1] \mathbf{A}^{\bullet} \stackrel{\mathrm{comp}}{\longrightarrow} \psi_{f}[-1] \mathbf{A}^{\bullet} \stackrel{\mathrm{can}}{\longrightarrow} \phi_{f}[-1] \mathbf{A}^{\bullet} \stackrel{[1]}{\longrightarrow} .
$$

The Wang triangle:

$$
\rightarrow j_{f}^{*}[-1] i_{f_{*}} i_{f}^{*} \mathbf{A}^{\bullet} \rightarrow \psi_{f}[-1] \mathbf{A}^{\bullet} \stackrel{\mathrm{id}-T_{f}}{\longrightarrow} \psi_{f}[-1] \mathbf{A}^{\bullet} \stackrel{[1]}{\longrightarrow} .
$$

The dual vanishing triangle:

$$
\rightarrow \phi_{f}[-1] \mathbf{A}^{\bullet} \stackrel{\operatorname{var}}{\longrightarrow} \psi_{f}[-1] \mathbf{A}^{\bullet} \stackrel{\mathrm{pmoc}}{\longrightarrow} j_{f}^{!}[1] \mathbf{A}^{\bullet} \stackrel{[1]}{\longrightarrow} .
$$

The dual Wang triangle:

$$
\rightarrow \psi_{f}[-1] \mathbf{A}^{\bullet} \stackrel{\mathrm{id}-T_{f}}{\longrightarrow} \psi_{f}[-1] \mathbf{A}^{\bullet} \rightarrow j_{f}^{!}[1] i_{f} i_{f}^{!} \mathbf{A}^{\bullet} \stackrel{[1]}{\longrightarrow} .
$$


There are equalities of natural maps

$$
\operatorname{var} \circ \text { can }=\mathrm{id}-T_{f} \quad \text { and } \quad \text { can } \circ \text { var }=\mathrm{id}-\widetilde{T}_{f} .
$$

The following theorem is a dual version of a generalization of the primary result of Lê in [6].

Theorem 3.5. Suppose that $\mathbf{P}^{\bullet}$ is a perverse sheaf on $X$, with free Abeian Morse modules. Let $\mathbf{x} \in V(f)$, and let $L$ be the restriction to $X$ of a generic affine linear form such that $L(\mathbf{x})=0$. Let $\check{m}_{\mathbf{x}}$ denote the inclusion of $\{\mathbf{x}\}$ into $V(f, L)$, let $\hat{m}_{\mathbf{x}}$ denote the inclusion of $\{\mathbf{x}\}$ into $V(f)$, let $\hat{L}$ denote the restriction of $L$ to $V(f)$, and let $j_{\hat{L}}$ denote the inclusion of $V(f, L)$ into $V(f)$.

Then, for all $k \geq 1$,

$$
H^{k+1}\left(\hat{m}_{\mathbf{x}}^{!} \psi_{f}[-1] \mathbf{P}^{\bullet}\right) \cong H^{k}\left(\check{m}_{\mathbf{x}}^{!} \psi_{f_{\mid V(L)}}[-1]\left(\mathbf{P}_{\left.\right|_{V(L)}}^{\bullet}[-1]\right)\right) .
$$

In addition, there is an exact sequence

$$
0 \rightarrow H^{0}\left(\hat{m}_{\mathbf{x}}^{!} \psi_{f}[-1] \mathbf{P}^{\bullet}\right) \rightarrow \mathbb{Z}^{\tau} \rightarrow H^{0}\left(\check{m}_{\mathbf{x}}^{!} \psi_{f_{\left.\right|_{V}(L)}}[-1]\left(\mathbf{P}_{\left.\right|_{V(L)}}[-1]\right)\right) \rightarrow H^{1}\left(\hat{m}_{\mathbf{x}}^{!} \psi_{f}[-1] \mathbf{P}^{\bullet}\right) \rightarrow 0
$$

where $\tau$ can be calculated as the intersection number of a general version of the relative polar curve with $V(f)$ at $\mathbf{x}$.

More generally, if $H^{c}$ denotes the intersection of $X$ with a generic affine linear subspace of codimension c in $\mathcal{U}$, then, for all $k \geq 1$

$$
H^{k+c}\left(\hat{m}_{\mathbf{x}}^{!} \psi_{f}[-1] \mathbf{P}^{\bullet}\right) \cong H^{k}\left(\check{m}_{\mathbf{x}}^{!} \psi_{f_{\left.\right|_{H^{c}}}}[-1]\left(\mathbf{P}_{\left.\right|_{H^{c}}}^{\bullet}[-c]\right)\right) \text {. }
$$

Proof. Theorem 4.1 of [13] implies that we have an isomorphism

$$
H^{*}\left(\check{m}_{\mathbf{x}}^{!} \psi_{\hat{L}}[-1] \psi_{f}[-1] \mathbf{P}^{\bullet}\right) \cong H^{*}\left(\check{m}_{\mathbf{x}}^{!} \psi_{f_{\left.\right|_{V(L)}}}[-1]\left(\mathbf{P}_{\left.\right|_{V(L)}}^{\bullet}[-1]\right)\right)
$$

The dual vanishing triangle for $\hat{L}$, applied to $\psi_{f}[-1] \mathbf{P}^{\bullet}$, yields

$$
\rightarrow \phi_{\hat{L}}[-1] \psi_{f}[-1] \mathbf{P}^{\bullet} \rightarrow \psi_{\hat{L}}[-1] \psi_{f}[-1] \mathbf{P}^{\bullet} \rightarrow j_{\hat{L}}^{!}[1] \psi_{f}[-1] \mathbf{P}^{\bullet} \stackrel{[1]}{\longrightarrow} .
$$

Applying $\check{m}_{\mathbf{x}}^{!}$, we have a distinguished triangle

$$
\rightarrow \check{m}_{\mathbf{x}}^{!} \phi_{\hat{L}}[-1] \psi_{f}[-1] \mathbf{P}^{\bullet} \rightarrow \check{m}_{\mathbf{x}}^{!} \psi_{\hat{L}}[-1] \psi_{f}[-1] \mathbf{P}^{\bullet} \rightarrow \hat{m}_{\mathbf{x}}^{!}[1] \psi_{f}[-1] \mathbf{P}^{\bullet} \stackrel{[1]}{\longrightarrow} .
$$

Now, for generic $L, \mathbf{x}$ is an isolated point in the support of the perverse sheaf $\phi_{\hat{L}}[-1] \psi_{f}[-1] \mathbf{P}^{\bullet}$. Hence, its cohomology is concentrated in degree 0 , and Corollary 4.6 .3 of [13] tells us that

$$
H^{0}\left(\check{m}_{\mathbf{x}}^{!} \phi_{\hat{L}}[-1] \psi_{f}[-1] \mathbf{P}^{\bullet}\right) \cong H^{0}\left(\check{m}_{\mathbf{x}}^{*} \phi_{\hat{L}}[-1] \psi_{f}[-1] \mathbf{P}^{\bullet}\right)
$$

is $\mathbb{Z}^{\tau}$, where $\tau$ is intersection number of a general version of the relative polar curve with $V(f)$ at $\mathbf{x}$.

The first two statements of the theorem now follow from taking the long exact cohomology sequence of $(*)$ our last distinguished triangle, and using $(\dagger)$ and $(\ddagger)$. The final statement follows from the first statement by induction. 


\section{$A^{\bullet}$-critical points}

We want critical points, with respect to a complex $\mathbf{A}^{\bullet}$, to be places where the hypercohomology of the fibers of $f$ changes.

Definition 3.6. Suppose that $\mathbf{A}^{\bullet} \in D_{c}^{b}(X)$. We say that $\mathbf{p} \in X$ is an $\mathbf{A}^{\bullet}$-critical point of $f$ if and only if $H^{*}\left(\phi_{f-f(\mathbf{p})} \mathbf{A}^{\bullet}\right) \neq 0$. We let $\Sigma_{\mathbf{A}} \bullet f$ denote the set of $\mathbf{A}^{\bullet}$-critical points of $f$.

In the special case where $\mathbf{A}^{\bullet}$ is the constant sheaf, or a shift of it, we simply write $\Sigma_{\mathbb{Z}} f$, rather than $\Sigma_{\mathbb{Z}_{X}} f$.

The following properties are well-known.

\section{Theorem 3.7.}

1. Suppose that $\mathfrak{S}$ is a Whitney stratification of $X$, with respect to which $\mathbf{A}^{\bullet}$ is constructible. Then, $\Sigma_{\mathbf{A}} \bullet f$ is contained in the stratified critical locus, i.e., $\Sigma_{\mathbf{A}} \bullet f \subseteq \bigcup_{S \in \mathfrak{S}} \Sigma\left(f_{\left.\right|_{S}}\right)$.

2. As stratified critical values are locally isolated, $\mathbf{A}^{\bullet}$-critical values are locally isolated, i.e., near a point $\mathbf{x} \in X, \Sigma_{\mathbf{A}} \bullet f \subseteq V(f-f(\mathbf{x}))$. Thus, near $\mathbf{x} \in V(f-f(\mathbf{x}))$,

$$
\overline{\Sigma_{\mathbf{A}} \cdot f}=\operatorname{supp} \phi_{f-f(\mathbf{x})} \mathbf{A}^{\bullet} .
$$

3. Suppose that $\mathbf{P}^{\bullet}$ is a perverse sheaf on $X, \mathbf{x} \in V(f)$, and let $s:=\operatorname{dim}_{\mathbf{x}} \Sigma_{\mathbf{P}} \bullet f$. Then, unless $-s \leq k \leq 0$, $H^{k}\left(\hat{m}_{\mathbf{x}}^{*} \phi_{f}[-1] \mathbf{P}^{\bullet}\right)=0$ and, unless $0 \leq k \leq s, H^{k}\left(\hat{m}_{\mathbf{x}}^{!} \phi_{f}[-1] \mathbf{P}^{\bullet}\right)=0$.

4. Suppose that $\mathbf{P}^{\bullet}$ is a perverse sheaf on $X, \mathbf{x} \in V(f)$, and let $L$ be the restriction to $X$ of a generic affine linear form such that $L(\mathbf{x})=0$. Then, $\operatorname{dim}_{\mathbf{x}} \Sigma_{\mathbf{P}} \cdot L=0$, and so

$$
\hat{m}_{\mathbf{x}}^{*} \phi_{L}[-1] \mathbf{P}^{\bullet} \cong \hat{m}_{\mathbf{x}}^{!} \phi_{L}[-1] \mathbf{P}^{\bullet}
$$

has possibly non-zero cohomology only in degree 0.

Suppose that $X$ is a local complete intersection of pure dimension $(n+1)$, and $\mathbf{P}^{\bullet}=\mathbb{Z}_{X}^{\bullet}[n+1]$. Then, (4) above is a weak form of the fact that the complex link of a point has the homotopy-type of a bouquet of $n$-spheres.

The next result is well-known in the classical situation. In the general case, it follows in the same way as the classical case: from the fact that $L$ may be chosen so that the intersection of $V(L)$ with the relative polar of $(f, L)$ with respect to $\mathbf{A}^{\bullet}$ is an isolated point. See [13].

Proposition 3.8. Let $\mathrm{x} \in V(f)$, and let $L$ be the restriction to $X$ of a generic affine linear form such that $L(\mathbf{x})=0$. Then, in an open neighborhood of $\mathbf{x}$,

$$
\left(\overline{\Sigma_{\left.\mathbf{A} \cdot\right|_{V(L)}}\left(f_{\left.\right|_{V(L)}}\right)}\right) \backslash\{\mathbf{x}\}=\left(V(L) \cap \overline{\Sigma_{\mathbf{A} \bullet} f}\right) \backslash\{\mathbf{x}\} .
$$

In particular, if $\operatorname{dim}_{\mathbf{x}} \Sigma_{\mathbf{A}} \cdot f \geq 1$, then

$$
\operatorname{dim}_{\mathbf{x}} \Sigma_{\left.\mathbf{A} \cdot\right|_{V(L)}}\left(f_{\left.\right|_{V(L)}}\right)=-1+\operatorname{dim}_{\mathbf{x}} \Sigma_{\mathbf{A}} \cdot f .
$$




\section{The variation map}

The natural map $\phi_{f}[-1] \stackrel{\mathrm{var}}{\longrightarrow} \psi_{f}[-1]$ in the dual vanishing triangle is called the variation map, and we will now explain its relationship to the classical variation map between the cohomology of the Milnor fiber and the Milnor fiber modulo its boundary.

Suppose that $\mathbf{p} \in V(f)$ and that $\operatorname{dim}_{\mathbf{p}} \Sigma_{\mathbf{A}} \bullet f=0$. Then, since $\mathbf{p}$ is an isolated point in the support of $\phi_{f}[-1] \mathbf{A}^{\bullet}$,

$$
\hat{m}_{\mathbf{p}}^{*} \phi_{f}[-1] \mathbf{A}^{\bullet} \cong \hat{m}_{\mathbf{p}}^{!} \phi_{f}[-1] \mathbf{A}^{\bullet},
$$

and so we have a morphism $\gamma_{\mathbf{p}}$ defined by

$$
\hat{m}_{\mathbf{p}}^{*} \psi_{f}[-1] \mathbf{A}^{\bullet} \stackrel{\hat{m}_{\mathbf{p}}^{*}(\operatorname{can})}{\longrightarrow} \hat{m}_{\mathbf{p}}^{*} \phi_{f}[-1] \mathbf{A}^{\bullet} \cong \hat{m}_{\mathbf{p}}^{!} \phi_{f}[-1] \mathbf{A}^{\bullet} \stackrel{\hat{m}_{\mathbf{p}}^{\prime}(\operatorname{var})}{\longrightarrow} \hat{m}_{\mathbf{p}}^{!} \psi_{f}[-1] \mathbf{A}^{\bullet} .
$$

Recalling the natural map $\hat{m}_{\mathbf{p}}^{!} \psi_{f}[-1] \mathbf{A}^{\bullet} \stackrel{\tau_{\mathbf{p}}}{\longrightarrow} \hat{m}_{\mathbf{p}}^{*} \psi_{f}[-1] \mathbf{A}^{\bullet}$, we have

$$
\tau_{\mathbf{p}} \circ \gamma_{\mathbf{p}}=\mathrm{id}-\hat{m}_{\mathbf{p}}^{*} T_{f} \quad \text { and } \quad \gamma_{\mathbf{p}} \circ \tau_{\mathbf{p}}=\mathrm{id}-\hat{m}_{\mathbf{p}}^{!} T_{f} .
$$

On the level of cohomology, this gives us: if $\mathbf{p} \in V(f)$ and $\operatorname{dim}_{\mathbf{p}} \Sigma_{\mathbf{A}} \bullet f=0$, then there are well-defined variation maps:

$$
\mathbb{H}^{k-1}\left(F_{f, \mathbf{p}} ; \mathbf{A}^{\bullet}\right) \stackrel{\nu_{\mathbf{p}}^{k-1}}{\longrightarrow} \mathbb{H}^{k-1}\left(F_{f, \mathbf{p}}, \partial F_{f, \mathbf{p}} ; \mathbf{A}^{\bullet}\right)
$$

such that, given the canonical maps

$$
\mathbb{H}^{k-1}\left(F_{f, \mathbf{p}}, \partial F_{f, \mathbf{p}} ; \mathbf{A}^{\bullet}\right) \stackrel{\tau_{\mathbf{p}}^{k-1}}{\longrightarrow} \mathbb{H}^{k-1}\left(F_{f, \mathbf{p}} ; \mathbf{A}^{\bullet}\right),
$$

we have

$$
\mathbb{H}^{k-1}\left(F_{f, \mathbf{p}}, \partial F_{f, \mathbf{p}} ; \mathbf{A}^{\bullet}\right) \stackrel{\nu_{\mathbf{p}}^{k-1} \circ \tau_{\mathbf{p}}^{k-1}=\mathrm{id}-T_{f, \mathbf{p}}^{k-1}}{\longrightarrow} \mathbb{H}^{k-1}\left(F_{f, \mathbf{p}}, \partial F_{f, \mathbf{p}} ; \mathbf{A}^{\bullet}\right),
$$

and

$$
\mathbb{H}^{k-1}\left(F_{f, \mathbf{p}} ; \mathbf{A}^{\bullet}\right) \stackrel{\tau_{\mathbf{p}}^{k-1} \circ \nu_{\mathbf{p}}^{k-1}=\mathrm{id}-T_{f, \mathbf{p}}^{k-1}}{\longrightarrow} \mathbb{H}^{k-1}\left(F_{f, \mathbf{p}} ; \mathbf{A}^{\bullet}\right)
$$

\section{Results for local complete intersections}

Our goal in this section is to see what the derived category results from the previous section tell us about the classical objects - the Milnor fiber, the real link, and the complex link - that we discussed in Section 2. All of the results which appear here are stated in completely classical terms, though the proofs go through the derived category.

Throughout this section, $X$ will denote a purely $(n+1)$-dimensional local complete intersection. For convenience, we assume that $\mathbf{0} \in X$, that $X$ is locally embedded in an open subset $\mathcal{U}$ of $\mathbb{C}^{N}$, and that $\mathbf{0}$ is a singular point of $X$ (for otherwise, everything here follows trivially from known results). We let $f:(X, \mathbf{0}) \rightarrow(\mathbb{C}, 0)$ be a complex analytic function. Finally, whenever we write ordinary cohomology, we mean with integral coefficients. 
Note that, if $f$ vanishes identically on some irreducible components of $X$, the Milnor fiber of $f$ is the same as the Milnor fiber of $f$ restricted to $\overline{X \backslash V(f)}$. Consequently, we will assume that $f$ does not vanish identically on any irreducible component of $X$ which contains the origin.

In the proofs, $\mathbf{P}^{\bullet}$ will denote the perverse sheaf $\mathbb{Z}_{X}^{\bullet}[n+1]$; by Theorem ??, $\mathcal{D} \mathbf{P}^{\bullet}$ is perverse, with free Abelian Morse modules, and is isomorphic to $\mathbb{Z}_{X \backslash \Sigma X}^{\bullet}[n+1]$ when restricted to $X \backslash \Sigma X$.

As in the introduction, we let $d:=\operatorname{dim}_{0} \Sigma X$. If $d=0$, we define $d_{f}:=0$; otherwise, we define $d_{f}:=\operatorname{dim}_{\mathbf{0}} \overline{\Sigma X \backslash V(f)}$. We let $s_{f}:=\operatorname{dim}_{\mathbf{0}} \Sigma_{\mathbb{Z}} f$. If $\mathbf{0} \notin \Sigma_{\mathbb{Z}} f$, we set $s_{f}=-\infty$.

Theorem 4.1. The reduced cohomology $\widetilde{H}^{k}\left(F_{f, \mathbf{0}}\right)=0$, except, possibly, when $n-s_{f} \leq k \leq n$. Furthermore, $\widetilde{H}^{n-s_{f}}\left(F_{f, 0}\right)$ is free Abelian.

Suppose that we are in the special case where $s_{f}=0$. Then, the reduced cohomology $\widetilde{H}^{k}\left(F_{f, \mathbf{0}}\right)$ equals zero, except, possibly, when $k=n$. In addition, $\operatorname{rank} \widetilde{H}^{n}\left(F_{f, \mathbf{0}}\right) \geq \operatorname{rank} \widetilde{H}^{n}\left(\mathbb{L}_{X, \mathbf{0}}\right)$.

Proof. The condition that $s_{f}=\operatorname{dim}_{\mathbf{0}} \Sigma_{\mathbb{Z}} f$ is equivalent to $s_{f}=\operatorname{dim}_{0} \operatorname{supp} \phi_{f}[-1] \mathbf{P}^{\bullet}$. As $\phi_{f}[-1] \mathbf{P}^{\bullet}$ is perverse, this implies that the only possibly non-zero stalk cohomology is in degrees $j$ such that $-s_{f} \leq j \leq 0$, i.e., if $j$ is not in this range, then

$$
H^{j}\left(\phi_{f}[-1] \mathbb{Z}_{X}^{\bullet}[n+1]\right)_{\mathbf{0}} \cong H^{j+n}\left(\phi_{f} \mathbb{Z}_{X}^{\bullet}\right)_{\mathbf{0}} \cong \widetilde{H}^{j+n}\left(F_{f, \mathbf{0}}\right)=0 .
$$

Lê's result tells us not just that $\mathbf{P}^{\bullet}$ is a perverse, but also that all of the Morse modules are free Abelian. Consequently, the Morse modules of $\phi_{f}[-1] \mathbf{P}^{\bullet}$ are free Abelian. It follows that $\widetilde{H}^{n-s_{f}}\left(F_{f, \mathbf{0}}\right)$ is free Abelian. Finally, that the rank of $H^{n}\left(F_{f, \mathbf{0}}\right)$ is at least the rank of $\widetilde{H}^{n}\left(\mathbb{L}_{X, \mathbf{0}}\right)$ is immediate from Theorem 5.3 of [11].

Remark 4.2. The first statement above can be found in Example 6.0.12 of [15]. The second statement also follows from Schürmann's work in Chapter 6 of [15].

The result from Theorem 5.3 of [11] is more general and more precise than the statement in Theorem 4.1, but, of course, that makes the statement harder to read.

There are also similar, more refined, results on the level of homotopy-type by Siersma [16] and Tibăr [17], under the strictly stronger hypothesis that $f$ has a stratified isolated critical point $\mathbf{0}$.

Theorem 4.3. $H^{k}\left(F_{f, \mathbf{0}}, \partial F_{f, \mathbf{0}}\right)=0$ unless $k=2 n$ or $n \leq k \leq n+\max \left\{s_{f}, d_{f}\right\}$. In addition, $H^{k}\left(F_{f, \mathbf{0}}, \partial F_{f, \mathbf{0}}\right)$ is free Abelian when $k=n$ and, if $d_{f} \neq n$, when $k=2 n$.

Proof. Using notation from the previous section, we need to look at

$$
H^{j}\left(\hat{m}_{\mathbf{0}}^{!} \psi_{f}[-1] \mathbf{P}^{\bullet}\right) \cong H^{j+n}\left(F_{f, \mathbf{0}}, \partial F_{f, \mathbf{0}}\right),
$$

and show that it is 0 , unless $j=n$ or $0 \leq j \leq \max \left\{s_{f}, d_{f}\right\}$. As $\psi_{f}[-1] \mathbf{P}^{\bullet}$ is supported on a set of dimension at most $n, H^{j}\left(\hat{m}_{\mathbf{0}}^{!} \psi_{f}[-1] \mathbf{P}^{\bullet}\right)$ unless $0 \leq j \leq n$. Thus, what we need to show is that $H^{j}\left(\hat{m}_{\mathbf{0}}^{!} \psi_{f}[-1] \mathbf{P}^{\bullet}\right)=0$ if $\max \left\{s_{f}, d_{f}\right\}+1 \leq j \leq n-1$.

- $d_{f}=0$ case: 
If $d_{f}=0$, then $F_{f, 0}$ is a smooth manifold with boundary, and Poincaré-Lefschetz duality tells us that $H^{k}\left(F_{f, \mathbf{0}}, \partial F_{f, \mathbf{0}}\right)$ is isomorphic to $H_{2 n-k}\left(F_{f, \mathbf{0}}\right)$. The result now follows from Theorem 4.1 and the Universal Coefficient Theorem.

- $d_{f}$ arbitrary case:

Let $H^{d_{f}}$ be the intersection of $X$ with a generic $d_{f}$-codimensional linear subspace in $\mathbb{C}^{N}$. Then, $H^{d_{f}}$ is a purely $\left(n+1-d_{f}\right)$-dimensional local complete intersection, and $g:=f_{\left.\right|_{H^{d}}}$ is a function such that $s_{g}=\max \left\{0, s-d_{f}\right\}$ and $d_{g}=0$.

By the previous case, we have that $H^{j}\left(\hat{m}_{\mathbf{0}}^{!} \psi_{g}[-1]\left(\mathbf{P}_{\left.\right|_{H^{d} f}}\left[-d_{f}\right]\right)\right)=0$ if $\max \left\{0, s_{f}-d_{f}\right\}+1 \leq j \leq n-d_{f}-1$. That $H^{j}\left(\hat{m}_{\mathbf{0}}^{!} \psi_{f}[-1] \mathbf{P}^{\bullet}\right)=0$ if $\max \left\{s_{f}, d_{f}\right\}+1 \leq j \leq n-1$ now follows immediately from Theorem 3.5. That $H^{n}\left(F_{f, \mathbf{0}}, \partial F_{f, \mathbf{0}}\right)$ is free Abelian also follows from Theorem 3.5 , since $H^{0}\left(\hat{m}_{\mathbf{x}}^{!} \psi_{f}[-1] \mathbf{P}^{\bullet}\right)$ injects into $\mathbb{Z}^{\tau}$. That $H^{2 n}\left(F_{f, \mathbf{0}}, \partial F_{f, \mathbf{0}}\right)$ is free Abelian also follows immediately from the $d_{f}=0$ case and the isomorphisms in Theorem 3.5, provided $n-d_{f} \geq 1$.

If $L$ is the restriction of a generic linear form to $X$, then $s_{L} \leq 0, d_{L}=d$, and $F_{L, 0}$ is the complex link $\mathbb{L}_{X, \mathbf{0}}$. Applying the theorem above, we immediately obtain:

Corollary 4.4. The relative cohomology $H^{k}\left(\mathbb{L}_{X, \mathbf{0}}, \partial \mathbb{L}_{X, \mathbf{0}}\right)=0$ unless $k=2 n$ or $n \leq k \leq n+d$. In addition, $H^{k}\left(\mathbb{L}_{X, \mathbf{0}}, \partial \mathbb{L}_{X, \mathbf{0}}\right)$ is free Abelian when $k=n$ and, if $d \neq n$, when $k=2 n$.

Taking the stalk cohomology of the Wang triangle and the costalk cohomology of the dual Wang triangle (see the previous section), both at the origin, and combining this with Theorem 4.1 and Theorem 4.3, we immediately obtain:

Theorem 4.5. There are long exact sequences, the Wang sequence and dual Wang sequence:

$$
\cdots \rightarrow H^{k-1}\left(F_{f, \mathbf{0}}\right) \rightarrow H^{k}\left(K_{X, \mathbf{0}} \backslash K_{V(f), \mathbf{0}}\right) \rightarrow H^{k}\left(F_{f, \mathbf{0}}\right) \stackrel{\text { id }-T_{f, \mathbf{0}}}{\longrightarrow} H^{k}\left(F_{f, \mathbf{0}}\right) \rightarrow H^{k+1}\left(K_{X, \mathbf{0}} \backslash K_{V(f), \mathbf{0}}\right) \rightarrow \cdots
$$

and

$$
\begin{aligned}
\cdots \rightarrow H^{k-1}\left(F_{f, \mathbf{0}}, \partial F_{f, \mathbf{0}}\right) \rightarrow H^{k}\left(K_{X, \mathbf{0}}, K_{V(f), \mathbf{0}}\right) \rightarrow H^{k}\left(F_{f, \mathbf{0}}, \partial F_{f, \mathbf{0}}\right) \stackrel{\mathrm{id}-T_{f, \mathbf{0}}}{\longrightarrow} \\
H^{k}\left(F_{f, \mathbf{0}}, \partial F_{f, \mathbf{0}}\right) \rightarrow H^{k+1}\left(K_{X, \mathbf{0}}, K_{V(f), \mathbf{0}}\right) \rightarrow \cdots .
\end{aligned}
$$

Remark 4.6. Note that, if $X$ itself has a non-isolated singularity at $\mathbf{0}$, then $K_{X, \mathbf{0}}$ and $F_{f, \mathbf{0}}$ are not manifolds, and the second long exact sequence above is not obtained via Poincaré-Lefschetz duality from the first one.

From Theorem 4.5 and Theorem 4.3, we immediately conclude:

Corollary 4.7. The integral cohomology $H^{k}\left(K_{X, \mathbf{0}} \backslash K_{V(f), \mathbf{0}}\right)=0$ except, possibly, when $k=0,1$ or $n-s_{f} \leq$ $k \leq n+1$.

The integral relative cohomology $H^{k}\left(K_{X, \mathbf{0}}, K_{V(f), \mathbf{0}}\right)=0$ except, possibly, when $k=2 n, 2 n+1$ or $n \leq$ $k \leq n+\max \left\{s_{f}, d_{f}\right\}+1$. 
By taking $f$ to be the restriction of a generic linear form, we obtain:

Corollary 4.8. Let $H$ denote e generic hyperplane in the ambient affine space. Then, the integral cohomology $H^{k}\left(K_{X, \mathbf{0}} \backslash K_{X \cap H, \mathbf{0}}\right)=0$ except, possibly, when $k=0,1$ or $n \leq k \leq n+1$.

The integral relative cohomology $H^{k}\left(K_{X, \mathbf{0}}, K_{X \cap H, \mathbf{0}}\right)=0$ except, possibly, when $k=2 n, 2 n+1$ or $n \leq$ $k \leq n+d+1$.

Theorem 4.9. Suppose that $n \geq 1$ and $s_{f}=0$. Let $\omega$ denote the map from $H^{n}\left(F_{f, \mathbf{0}}, \partial F_{f, \mathbf{0}}\right)$ to $H^{n}\left(F_{f, \mathbf{0}}\right)$ induced by the inclusion $\left(F_{f, \mathbf{0}}, \emptyset\right) \hookrightarrow\left(F_{f, \mathbf{0}}, \partial F_{f, \mathbf{0}}\right)$.

Then:

1. There is well-defined variation map

$$
\text { var : } H^{n}\left(F_{f, \mathbf{0}}\right) \rightarrow H^{n}\left(F_{f, \mathbf{0}}, \partial F_{f, \mathbf{0}}\right)
$$

such that $\operatorname{var} \circ \omega=\mathrm{id}-T_{f}$ on $H^{n}\left(F_{f, \mathbf{0}}, \partial F_{f, \mathbf{0}}\right)$ and $\omega \circ \operatorname{var}=\mathrm{id}-T_{f}$ on $H^{n}\left(F_{f, \mathbf{0}}\right)$.

2. There is an exact sequence

$$
0 \rightarrow H^{n}\left(K_{X, \mathbf{0}}\right) \rightarrow H^{n}\left(F_{f, \mathbf{0}}\right) \stackrel{\mathrm{var}}{\longrightarrow} H^{n}\left(F_{f, \mathbf{0}}, \partial F_{f, \mathbf{0}}\right) \rightarrow H^{n+1}\left(K_{X, \mathbf{0}}\right) \rightarrow 0 .
$$

In particular, $H^{n}\left(K_{X, \mathbf{0}}\right)$ injects into $H^{n}\left(F_{f, \mathbf{0}}\right)$, and var is an isomorphism if and only if $H^{n}\left(K_{X, \mathbf{0}}\right)=$ $H^{n+1}\left(K_{X, \mathbf{0}}\right)=0$.

3. For all $k \geq n+1, H^{k}\left(F_{f, \mathbf{0}}, \partial F_{f, \mathbf{0}}\right) \cong H^{k+1}\left(K_{X, \mathbf{0}}\right)$, and so, for all $k \geq n+1, H^{k}\left(\partial F_{f, \mathbf{0}}\right) \cong H^{k+2}\left(K_{X, \mathbf{0}}\right)$.

4. For all $k \leq n-1, \widetilde{H}^{k}\left(K_{X, 0}\right)=0$.

Proof. The existence of the variation map, satisfying Item (1), is immediate from our general categorical discussion in the previous section.

Now, since $s_{f}=0$ and $n \geq 1$, the map $H^{n}\left(F_{f, \mathbf{0}}\right) \stackrel{\text { can }}{\longrightarrow} \widetilde{H}^{n}\left(F_{f, \mathbf{0}}\right)$ is an isomorphism. In addition, since $s_{f}=0$,

and $H^{k}\left(\hat{m}_{\mathbf{0}}^{!} \phi_{f}[-1] \mathbf{P}^{\bullet}\right)=0$ for $k \neq 0$.

$$
\widetilde{H}^{n}\left(F_{f, \mathbf{0}}\right) \cong H^{0}\left(\hat{m}_{\mathbf{0}}^{*} \phi_{f}[-1] \mathbf{P}^{\bullet}\right) \cong H^{0}\left(\hat{m}_{\mathbf{0}}^{!} \phi_{f}[-1] \mathbf{P}^{\bullet}\right)
$$

If we apply $\hat{m}_{\mathbf{0}}$ to the dual vanishing triangle, we obtain the distinguished triangle

$$
\rightarrow \hat{m}_{\mathbf{0}}^{!} \phi_{f}[-1] \mathbf{P}^{\bullet} \stackrel{\mathrm{var}}{\longrightarrow} \hat{m}_{\mathbf{0}}^{!} \psi_{f}[-1] \mathbf{P}^{\bullet} \stackrel{\mathrm{pmoc}}{\longrightarrow} m_{\mathbf{0}}^{!}[1] \mathbf{P}^{\bullet} \stackrel{[1]}{\longrightarrow} .
$$

Taking the long exact cohomology sequence of this triangle immediately yields Items (2), (3), and (4) after one notes that

$$
H^{k}\left(m_{\mathbf{0}}^{!}[1] \mathbf{P}^{\bullet}\right) \cong H^{k+n+2}\left(B_{\epsilon}(\mathbf{0}) \cap X, S_{\epsilon}(\mathbf{0}) \cap X\right) \cong \widetilde{H}^{k+n+1}\left(K_{X, \mathbf{0}}\right)
$$

Applying this theorem to the restriction of a generic linear form, we conclude: 
Corollary 4.10. Suppose that $n \geq 1$, that $L$ is the restriction of a generic linear form to $X$, and that $H=V(L)$. Then:

1. There is well-defined variation map

$$
\operatorname{var}: H^{n}\left(\mathbb{L}_{X, \mathbf{0}}\right) \rightarrow H^{n}\left(\mathbb{L}_{X, \mathbf{0}}, \partial \mathbb{L}_{X, \mathbf{0}}\right)
$$

such that $\operatorname{var} \circ \omega=\mathrm{id}-T_{L}$ on $H^{n}\left(\mathbb{L}_{X, \mathbf{0}}, \partial \mathbb{L}_{X, \mathbf{0}}\right)$ and $\omega \circ \operatorname{var}=\mathrm{id}-T_{L}$ on $H^{n}\left(\mathbb{L}_{X, \mathbf{0}}\right)$.

2. There is an exact sequence

$$
0 \rightarrow H^{n}\left(K_{X, \mathbf{0}}\right) \rightarrow H^{n}\left(\mathbb{L}_{X, \mathbf{0}}\right) \stackrel{\text { var }}{\longrightarrow} H^{n}\left(\mathbb{L}_{X, \mathbf{0}}, \partial \mathbb{L}_{X, \mathbf{0}}\right) \rightarrow H^{n+1}\left(K_{X, \mathbf{0}}\right) \rightarrow 0 .
$$

In particular, $H^{n}\left(K_{X, \mathbf{0}}\right)$ injects into $H^{n}\left(\mathbb{L}_{X, \mathbf{0}}\right)$, and var is an isomorphism if and only if $H^{n}\left(K_{X, \mathbf{0}}\right)=$ $H^{n+1}\left(K_{X, \mathbf{0}}\right)=0$.

3. For all $k \geq n+1, H^{k}\left(\mathbb{L}_{X, \mathbf{0}}, \partial \mathbb{L}_{X, \mathbf{0}}\right) \cong H^{k+1}\left(K_{X, \mathbf{0}}\right)$, and so, for all $k \geq n+1$,

$$
H^{k}\left(K_{X \cap H, \mathbf{0}}\right) \cong H^{k}\left(\partial \mathbb{L}_{X, \mathbf{0}}\right) \cong H^{k+2}\left(K_{X, \mathbf{0}}\right) .
$$

Remark 4.11. That $H^{n}\left(K_{X, 0}\right)$ injects into $H^{n}\left(\mathbb{L}_{X, 0}\right)$, regardless of the dimension of the singular set of $X$ generalizes part of the result given by Dimca in Proposition 6.1.22 of [2], in which $d$ is required to be 0 .

Combining Item (3) of the previous corollary with Corollary 4.4, we obtain:

Corollary 4.12. The integral cohomology $H^{k}\left(K_{X, \mathbf{0}}\right)=0$, unless $k=0, k=2 n+1$, or $n \leq k \leq n+d+1$.

Example 4.13. Of course, it is natural to ask for an example where $d>0, n+d+1 \neq 2 n+1$ (i.e., $d \neq n$ ), and $H^{n+d+1}\left(K_{X, 0}\right) \neq 0$.

In fact, it is easy to produce such examples. Let $f: \mathbb{C}^{5} \rightarrow \mathbb{C}$ be given by $f(t, w, x, y, z)=w^{2}+x^{2}+y^{2}+z^{2}$ and let $X=V(f)$, i.e., $X$ is the product of an ordinary quadratic singularity in $\mathbb{C}^{4}$ with a complex line. Here, $n=3$ and $d=1$.

Then, by Item (3) of Corollary 4.10, and since $\mathbb{L}_{X, 0}$ is contractible,

$$
H^{n+d+1}\left(K_{X, \mathbf{0}}\right) \cong H^{5}\left(K_{X, \mathbf{0}}\right) \cong H^{4}\left(\mathbb{L}_{X, \mathbf{0}}, \partial \mathbb{L}_{X, \mathbf{0}}\right) \cong H^{3}\left(\partial \mathbb{L}_{X, \mathbf{0}}\right) \cong H^{3}\left(K_{X \cap V(t), \mathbf{0}}\right) .
$$

But $K_{X \cap V(t), \mathbf{0}}$ is the real link of the ordinary quadratic singularity $V\left(w^{2}+x^{2}+y^{2}+z^{2}\right)$ in $\mathbb{C}^{4}$. It is well-known that the monodromy on $\mathrm{H}_{3}$ of the corresponding Milnor fiber is the identity (since the Lefschetz number of the monodromy must be zero). Thus, using the Wang sequence on homology, we know that

$$
\mathbb{Z} \cong H_{3}\left(S_{\epsilon}^{7} \backslash K_{X \cap V(t), \mathbf{0}}\right) \cong H^{4}\left(S_{\epsilon}^{7}, K_{X \cap V(t), \mathbf{0}}\right) \cong H^{3}\left(K_{X \cap V(t), \mathbf{0}}\right) \cong H^{5}\left(K_{X, \mathbf{0}}\right) .
$$




\section{References}

[1] Dimca, A. Singularities and Topology of Hypersurfaces. Universitext. Springer-Verlag, 1992.

[2] Dimca, A. Sheaves in Topology. Universitext. Springer-Verlag, 2004.

[3] Goresky, M. and MacPherson, R. Stratified Morse Theory, volume 14 of Ergeb. der Math. SpringerVerlag, 1988.

[4] Hamm, H. . Lokale topologische Eigenschaften komplexer Räume. Math. Annalen, 191:235-252, 1971.

[5] Kashiwara, M. and Schapira, P. Sheaves on Manifolds, volume 292 of Grund. math. Wissen. SpringerVerlag, 1990.

[6] Lê, D. T. Calcul du Nombre de Cycles Évanouissants d'une Hypersurface Complexe. Ann. Inst. Fourier, Grenoble, 23:261-270, 1973.

[7] Lê, D. T. Some remarks on Relative Monodromy. In P. Holm, editor, Real and Complex Singularities, Oslo 1976, pages 397-404. Nordic Summer School/NAVF, 1977.

[8] Lê, D. T. Sur les cycles évanouissants des espaces analytiques. C. R. Acad. Sci. Paris, Sér. A-B, 288:A283-A285, 1979.

[9] Lê, D. T. and Kato, M. Vanishing cycles on analytic sets. Proc. Symp. on Alg. Analysis, R.I.M.S., Kyoto, 1975.

[10] Looijenga, E. Isolated Singular Points on Complete Intersections. Cambridge Univ. Press, 1984.

[11] Massey, D. A Little Microlocal Morse Theory. Math. Ann., 321:275-294, 2001.

[12] Massey, D. Singularities and Enriched Cycles. Pacific J. Math., 215, no. 1:35-84, 2004.

[13] Massey, D. Enriched Relative Polar Curves and Discriminants. Contemp. Math., 474:107-144, 2008.

[14] Milnor, J. Singular Points of Complex Hypersurfaces, volume 77 of Annals of Math. Studies. Princeton Univ. Press, 1968.

[15] Schürmann, J. Topology of Singular Spaces and Constructible Sheaves, volume 63 of Monografie Matematyczne. Birkhäuser, 2004.

[16] Siersma, D. A bouquet theorem for the Milnor fibre. Joun. Alg. Geom., 4:51-66, 1995.

[17] Tibăr, M. Bouquet Decomposition of the Milnor Fiber. Topology, 35:227-241, 1996. 